\title{
Study of Indium Tin Oxide Thin Films Deposited on Acrylics Substrates by Ion Beam Assisted Deposition Technique
}

\author{
Li-Jian Meng ${ }^{1,2, *}$, Erjun Liang ${ }^{3}$, Jinsong Ga0 ${ }^{4}$, \\ V. Teixeira ${ }^{2}$, and M. P. dos Santos ${ }^{5}$ \\ ${ }^{1}$ Departamento de Física, Instituto Superior de Engenharia do Porto, Rua Dr. António Bernardino de Almeida, \\ 431, 4200-072 Porto, Portugal \\ ${ }^{2}$ Centro de Física, Universidade do Minho, 4700 Braga, Portugal \\ ${ }^{3}$ School of Physical Science and Engineering, Zhengzhou University, Zhengzhou 450052, China \\ ${ }^{4}$ Center of Optical Technology, Changchun Institute of Optics, Fine Mechanics and Physics, \\ Chinese Academy of Sciences, Changchun 130033, China \\ ${ }^{5}$ CeFITec, Universidade Nova de Lisboa/Departamento de Física, Universidade de Évora, 7000, Portugal
}

\begin{abstract}
Indium tin oxide (ITO) thin films have been deposited onto acrylics (PMMA) substrates by ion beam assisted deposition technique at different oxygen flows. The structural, optical and electrical properties of the deposited films have been characterized by X-ray diffraction, transmittance, FTIR, ellipsometry and Hall effect measurements. The optical constants of the deposited films have been calculated by fitting the ellipsometric spectra. The effects of the oxygen flow on the properties of the deposited films have been studied. It has been found that $40 \mathrm{sccm}$ oxygen flow is an optimum value for getting the films with good transmittance and low electrical resistivity.
\end{abstract}

Keywords: ITO, Optical Properties, Thin Film, PMMA, Ion Beam Assisted Deposition, Indium Tin Oxide.

\section{INTRODUCTION}

Indium tin oxide (ITO) is a tin-doped $\operatorname{In}_{2} \mathrm{O}_{3}$ based $n$-type degenerate wide bandgap semiconductor. The degeneracy is caused by both oxygen vacancy and substitutional tin created during deposition. ITO material in thin film form has attracted much attention from both basic scientific research and industrial applications for many years because of its low resistivity, high transmittance in visible and near infrared regions, stable physical and chemical properties, as well as good adhesion to many kinds of substrates. ${ }^{1}$ It has been widely used in the optoelectronic area such as flat panel display, solar cell devices and organic light emitting diodes as transparent conducting electrode. ${ }^{2}$ ITO thin films can be deposited by many techniques such as evaporation, sputtering, chemical vapour deposition, laser ablation, sol-gel process, and ion beam assisted deposition..$^{3-10}$ However, in order to obtain ITO films with low resistivity and high transmittance, most of these deposition techniques require or a high substrate temperature or a post annealing treatment (over than

${ }^{*}$ Author to whom correspondence should be addressed. $\left.300{ }^{\circ} \mathrm{C}\right)$. Recently, the interest in deposition of ITO film in flexible optoelectronic devices has grown up. ${ }^{11}$ It is necessary for ITO films to be deposited at very low substrate temperature due to the poor thermal endurance of polymer substrates. Ion beam assisted deposition (IBAD) has been considered as a low temperature deposition technique and has been used to deposit ITO films. Several transparent organic substrates such as polycarbonate (PC), ${ }^{12}$ polypropylene adipate (PPA), ${ }^{13}$ polyimide (PI) ${ }^{3}$ polyethylene terepthalate $(\mathrm{PET})^{4}$ have been used to deposit ITO films. Many reports can be found on ITO films deposited onto PC and PET substrates. However, it is hardly to find the report on ITO films deposited onto polymethyl methacrylate (PMMA) substrate. In this study, the ITO films were deposited onto PMMA substrate at room temperature by ion beam assisted deposition technique. The effects of the oxygen flow on the structural, optical and electrical properties were reported.

\section{EXPERIMENTAL DETAILS}

ITO films were deposited onto the commercial acrylics (PMMA) substrates at room temperature by ion beam 
assisted deposition technique using a vacuum coater equipped with electron beam gun and a Kaufman ion source. ITO powder pellet with a composition of $90 \mathrm{wt} \%$ $\mathrm{In}_{2} \mathrm{O}_{3}$ and $10 \mathrm{wt} \% \mathrm{SnO}_{2}$ was used as the evaporation source material. A $120 \mathrm{~mm}$ diameters Kaufman ion source was used to generate oxygen ion beam. The oxygen gas flow was controlled by a mass flow controller. The deposition rate and the film thickness were monitored and controlled by a quartz crystal sensor which has been linked to e-beam power supply for automatic controlling. The nominal deposition rate and the thickness were preset at $0.2 \mathrm{~nm} / \mathrm{s}$ and $200 \mathrm{~nm}$, respectively. The substrate holder was rotated at a speed of 0.3 rounds/s. The angle between the incident oxygen ion beam and the normal of the substrate holder was fixed at $45^{\circ}$. Before the deposition, the chamber was evacuated until a pressure of $1 \times 10^{-3} \mathrm{~Pa}$. After that, the oxygen gas was introduced into the chamber. The oxygen flow was set to be 50, 40, 30 and $20 \mathrm{sccm}$, respectively. And the dynamic pressure in the chamber was about $2.9 \times 10^{-2}, 2.3 \times 10^{-2}, 1.8 \times 10_{\mathrm{g}}^{-2}$ and $1.4 \times 10^{-2} \mathrm{~Pa}$, respectively. For all deposition processes, the ion beam current, the accelerating voltage and the screen voltage were kept constants of $100 \mathrm{~mA}, 250 \mathrm{~V}$ and $300 \mathrm{~V}$.

The optical transmittance spectra of the films were recorded by Perklin-Elmer Lambda 900 UV/VIS/NIR spectrometer and the infrared reflectance was measured by Perklin-Elmer Spectrum GX at angle of incidence of $60^{\circ}$. The ellipsometric parameters IS and IC were measured using a Jobin-Yvon UVISEL ellipsometer acquired at $70.25^{\circ}$ of incidence angle over the spectral range $300-800 \mathrm{~nm}$ in steps of $5 \mathrm{~nm}$. The X-ray diffraction was done by SHIMADZU XRD-6000 performed between the $2 \theta$ values of $20^{\circ}-70^{\circ}$ with a step of $0.05^{\circ}$. Atomic force microscopy (AFM) measurements were made using equipment from Digital Instruments Veeco Metrology Group. The Hall effect was measured using Lake Shore 665 with a $5 \mathrm{kG}$ magnetic field intensity at room temperature.

\section{RESULTS AND DISCUSSION}

The X-ray diffraction measurements show that the films deposited at different oxygen flows are all amorphous. It means that the ion beam energy used in this work $(300 \mathrm{~V}$ screen voltage) is not enough to form the polycrystalline ITO film, although it is supposed that the additional heat energy and increased surface mobility of adatom by ion bombardment can result in polycrystalline ITO growth. ${ }^{14}$ In order to form the polycrystalline ITO films, it is necessary to increase the ion beam energy to a certain level. Our work has shown that the polycrystalline ITO films will be produced only when the screen voltage, which is related directly with ion beam energy, is higher than $500 \mathrm{~V} .{ }^{17}$ The specular transmittance of ITO films deposited at different oxygen flows are shown in Figure 1. It can be seen clearly

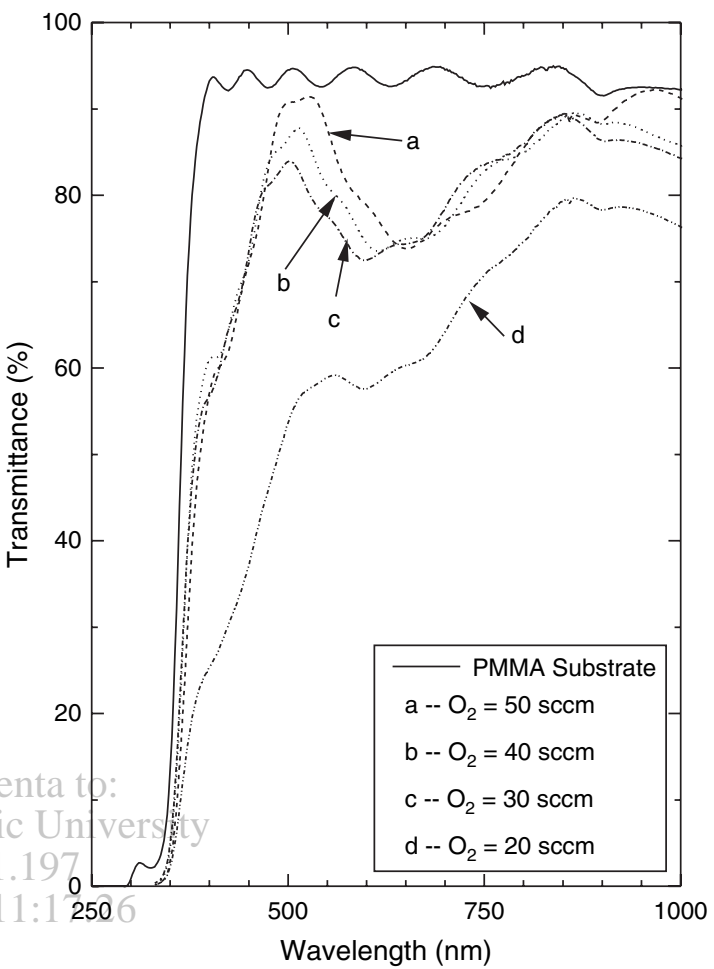

Fig. 1. Specular transmittance of ITO films deposited at different oxygen flows.

that the transmittance increases with oxygen flow. When $\mathrm{In}_{2} \mathrm{O}_{3}$ and $\mathrm{SnO}_{2}$ mixed oxides pellet are evaporated, the oxygen will be lost and a metal-like, brownish and less transparent film of lower oxide will be formed. It has been found that the ITO film deposited in this work without oxygen flow is opaque which indicates the importance of the oxygen ion bombardment during the deposition process for forming transparent ITO films. At low oxygen flow, the oxidation is incomplete and leads to a low transmittance. At high oxygen flow, the stoichiometric ITO film will be formed and results in a high transmittance. For comparison, the transmittance of bare PMMA substrate is also shown in Figure 1. The PMMA substrate used in this work has been treated to increase its surface hardness. The small fringes in the transmittance of PMMA substrate come from this treatment. This treatment also improves its transmittance. The transmittance of conventional PMMA substrate in the visible region is about $91 \% .{ }^{15}$ But the transmittance of our PMMA substrate in the visible region is about $95 \%$. The highest transmittance, $91 \%$ at $500 \mathrm{~nm}$ wavelength, has been reached for ITO films prepared at $50 \mathrm{sccm}$ oxygen flow.

For getting the optical constants (refractive index $n$ and extinction coefficient $k$ ) and the film thickness of the ITO films deposited at different oxygen flows, the ellipsometric measurements have been performed for all samples as shown in Figure 2. The ellipsometric spectra have been fitted using the Lorentz oscillator model (classical model). 


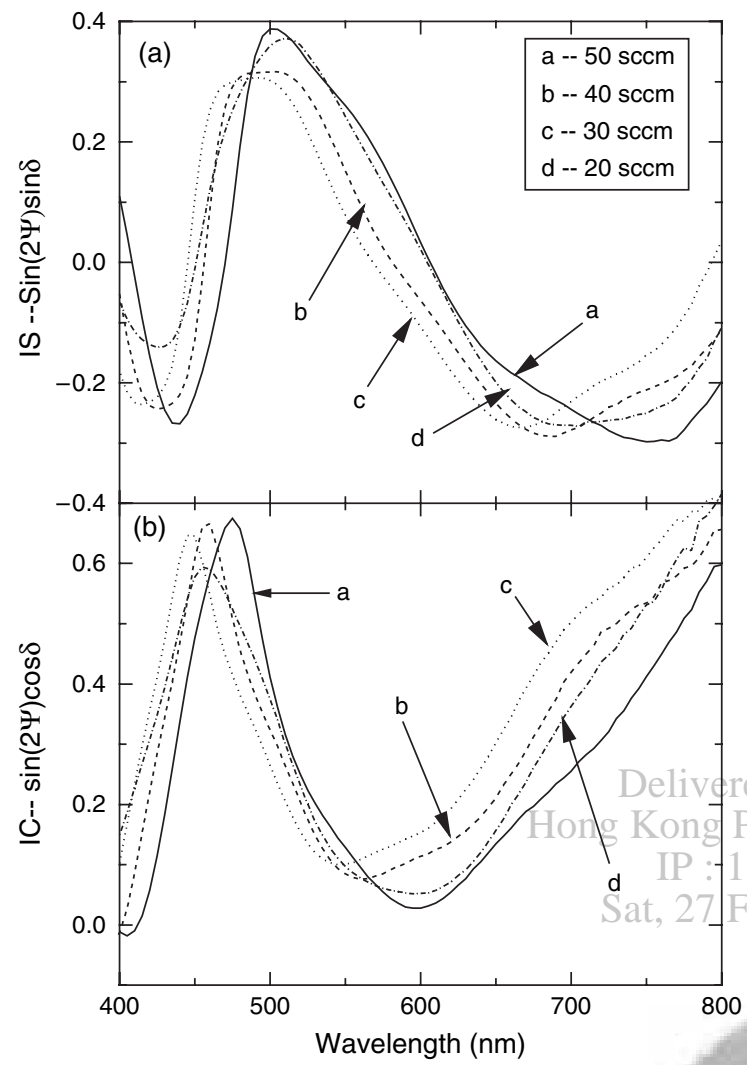

Fig. 2. Ellipsometric spectra of ITO films deposited at different oxygen flows, (a) IS; (b) IC.

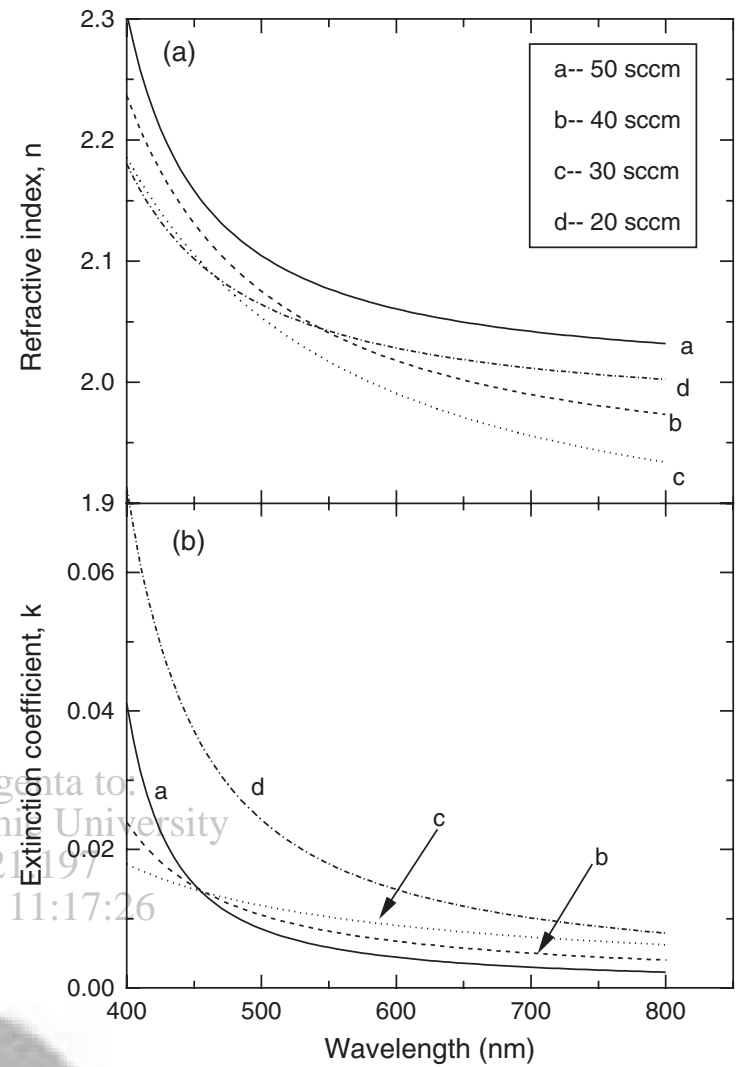

Fig. 3. (a) Refractive index and (b) extinction coefficient of ITO films deposited at different oxygen flows.

boundary scattering can be neglected. As the evaporation materials are same for all processes, the impurity in all the films should not have a big difference. Therefore, the main scattering source should be the oxygen vacancies in our ITO films. It is known that the film prepared at low oxygen flow has many oxygen vacancies. These oxygen vacancies supply the electrons for conductivity. But in the same time the light will also be scattered by these vacancies. As the oxygen flow is increased, the number of the oxygen vacancies goes down; the loss of the scattering light decreases and leads to a low extinction coefficient. It has been found that the film prepared at high oxygen flow has high refractive index. As the oxygen flow is increased,

Table I. Properties of ITO films deposited at different oxygen flows.

\begin{tabular}{lcccc}
\hline & PMMA1 & PMMA2 & PMMA3 & PMMA4 \\
\hline Oxygen flow (sccm) & 50 & 40 & 30 & 20 \\
$d(\mathrm{~nm})$ & 245 & 240 & 236 & 242 \\
$\varepsilon_{\infty}$ & 3.59 & 2.57 & 3.12 & 3.38 \\
$\varepsilon_{s}$ & 4.03 & 3.72 & 3.49 & 3.91 \\
$\omega_{t}$ & 3.58 & 4.26 & 4.79 & 3.90 \\
$\gamma_{0}$ & 0.12 & 0.12 & 0.14 & 0.42 \\
$\left.R_{(\Omega / \mathrm{square})} \Omega-\mathrm{cm}\right)$ & 150 & 27 & 25 & 67 \\
$\rho\left(\times 10^{-3} \mathrm{SS}\right)$ & 15.8 & 2.8 & 2.5 & 7.0 \\
$\mu\left(\mathrm{cm}^{2} / \mathrm{VS}\right)$ & 28.6 & 31.8 & 30.4 & 10.2 \\
$n\left(\times 10^{20} \mathrm{~cm}^{-3}\right)$ & 0.6 & 3.0 & 3.4 & 3.8 \\
\hline
\end{tabular}




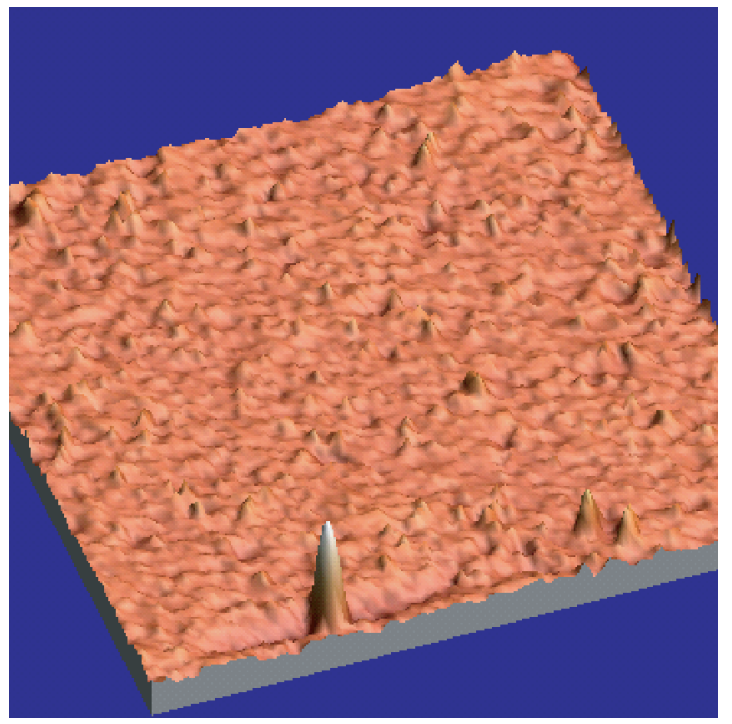

Fig. 4. AFM image of ITO film on PMMA substrate prepared at $40 \mathrm{sccm}$ oxygen flow.

Hong Kong Polyte

the ITO film approximates its stoichiometry and leads to an increase of the refractive index.

The electrical properties of ITO films deposited at different oxygen flows have been characterized by Hall effect measurement. The Hall mobility, electron concentration, sheet resistance and electrical resistivity of these ITO films

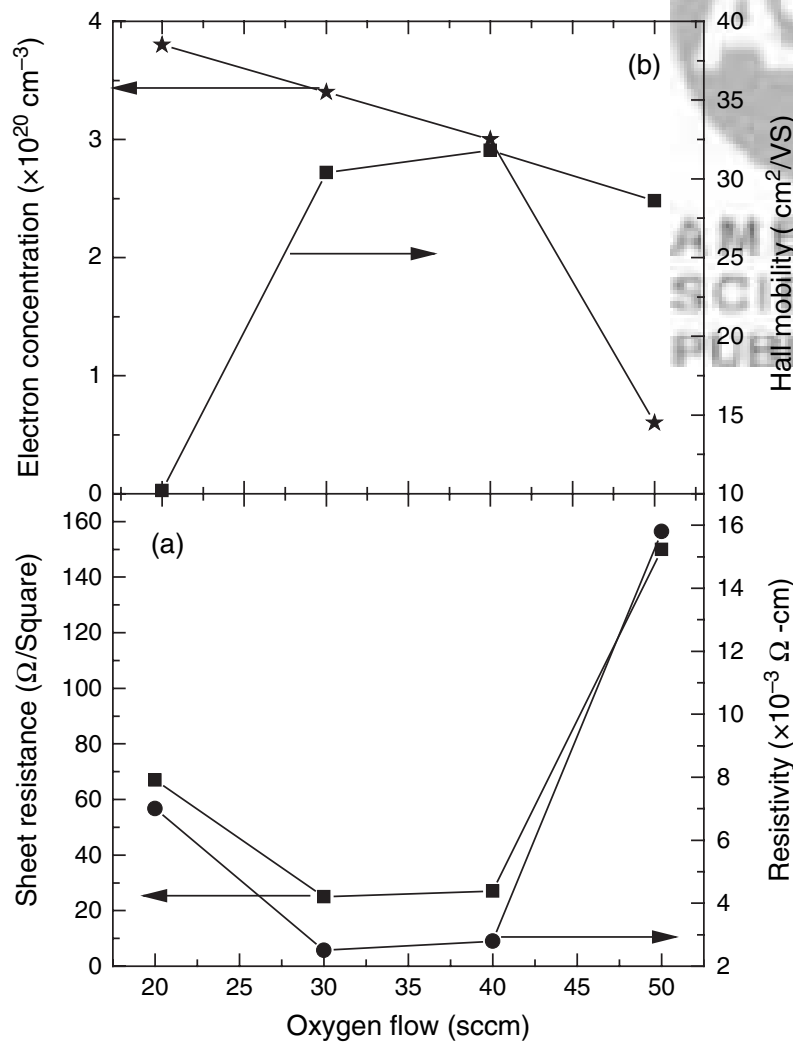

Fig. 5. (a) Sheet resistance and electrical resistivity and (b) electron concentration and Hall mobility with oxygen flow. have been given in Table I and plotted in Figure 5. It can be seen that ITO films prepared at 30-40 sccm oxygen flow have low resistivity. From Figure 5(b) it can be seen that the electron concentration decreases as the oxygen flow is increased. It is known that the one origin of the electron in ITO film is from the oxygen vacancies, as the oxygen flow is increased, the number of the oxygen vacancy decreases and results in the decreasing of the carrier concentration. Although the electron concentration is decreased with oxygen flow, the Hall mobility gets high value at $30-40 \mathrm{sccm}$ oxygen flow and then leads to a low resistivity.

The infrared reflectance spectra of ITO films deposited at different oxygen flows are given in Figure 6. The variation of the reflectance is related with the electrical properties of the films. Frank et al. ${ }^{16}$ showed that the infrared reflectance $R$ for high reflection level can be expressed by

$$
R=1-\frac{4 \varepsilon_{0} c_{0}}{e} \cdot \frac{1}{d n \mu}
$$

where $\varepsilon_{0}$ (the permittivity of electron in vacuum) $=8.85 \times$ $10^{-12}, \mathrm{c}_{0}$ (velocity of light in vacuum) $=3 \times 10^{8} \mathrm{~m} / \mathrm{s}$, $e($ electronic charge $)=1.6 \times 10^{-19} \mathrm{C}, n$ is the carrier concentration, $d$ is the film thickness and $\mu$ is the carrier mobility.

According to this equation, as ITO films deposited at different oxygen flows have a similar thickness (Table I), the film which has both high electron concentration and Hall mobility will has high reflectance. Our experimental results are agreeable with it. The films prepared at 30 and

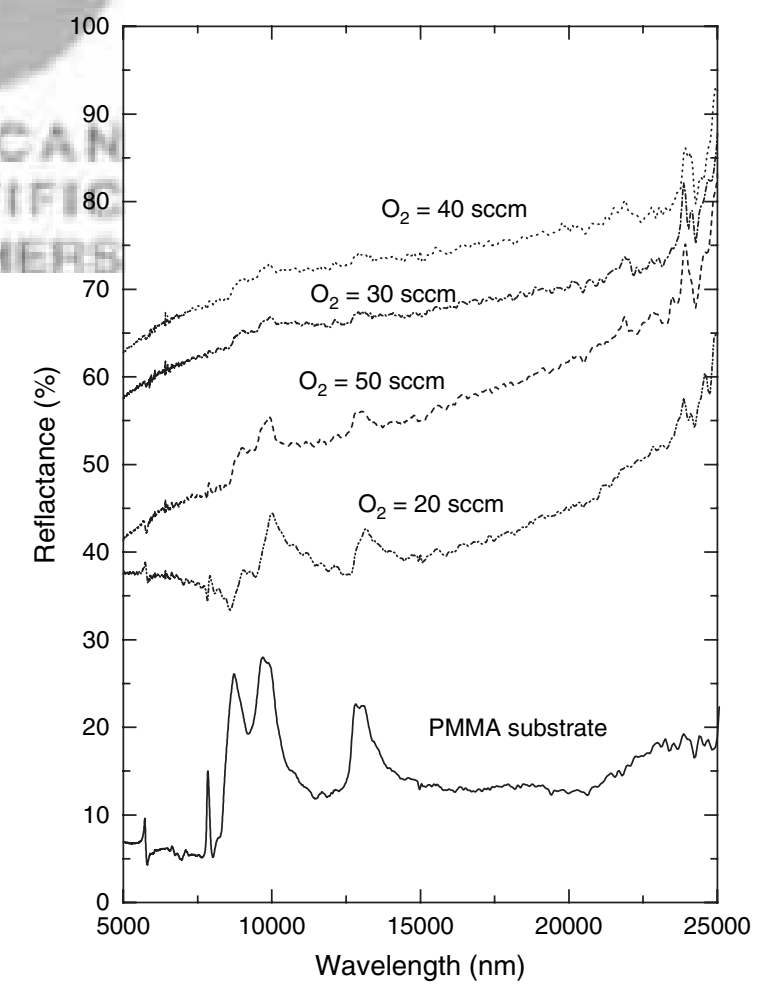

Fig. 6. FTIR spectra of ITO films deposited at different oxygen flows. 
$40 \mathrm{sccm}$ oxygen flows have high electron concentrations and Hall mobilities and give high reflectance as shown in Figure 6.

\section{CONCLUSIONS}

ITO films have been deposited onto PMMA substrates at room temperature using ion beam assisted deposition technique. The oxygen flow is varied from 20 to $50 \mathrm{sccm}$. All the films show amorphous structure. The film prepared at high oxygen flow gives high optical transmittance, high refractive index and low extinction coefficient. Although the maximum transmittance $(91 \%$ at $500 \mathrm{~nm})$ is obtained for ITO film deposited at $50 \mathrm{sccm}$ oxygen flow, it gives a high electrical resistivity $\left(15.8 \times 10^{-3} \Omega\right.$-cm). By considering both the transmittance and the electrical resistivity, It has been found that $40 \mathrm{sccm}$ oxygen flow is the optimum value for depositing ITO films. At this condition, the ITO films with $87 \%$ transmittance at $500 \mathrm{~nm}$ and $2.8 \times 10^{-3} \Omega$-cm electrical resistivity have been obtained. It shows that ion beam assisted deposition technique is a suitable technique to deposit high quality ITO films at low substrate temperature.

Acknowledgments: This work was carried out in the Centre of Optical Technology, Changchun Institute of Optics, fine Mechanics and Physics of Chinese Academy of Sciences during Li-Jian Meng's visit to the centre as a senior visiting researcher. Li-Jian Meng is thankful to the Fundação para a Ciência e a Tecanologia (Portugal) for providing a fellowship.

\section{References and Notes}

1. K. L. Chopra, S. Major, and D. K. Pandya, Thin Solid Films 102, 1 (1983).

2. S. A. Carter, M. Angelopoulos, S. Karg, P. J. Brock, and J. C. Scott, Appl. Phys. Lett. 70, 2067 (1997).

3. J. Ma, D. Zhang, J. Zhao, C. Tan, T. Yang, and H. Ma, Appl. Surf. Sci. 151, 239 (1999).

4. C. Guillén and J. Herrero, Thin Solid Films 480, 129 (2005).

5. P. K. Biswas, A. De, N. C. Pramanik, P. K. Chakraborty, K. Ortner, V. Hock, and S. Korder, Mater. Lett. 57, 2327 (2003).

6. T. K. Yong, T. Y. Tou, and B. S. Teo, Appl. Surf. Sci. 248, 388 (2005).

7. D. Kim, J. Non-Crystalline Solids 331, 41 (2003).

8. A. S. A. C. Diniz and C. J. Kiely, Renewable Energy 29, 2037 (2004).

9. M. Gilo, R. Dahan, and N. Croitoru, Opt. Eng. 38, 953 (1999).

10. C. Liu, T. Matsutani, T. Asanuma, and M. Kiuchi, Nuclear Instruiments and Methods in Physica Research B 206, 348 (2003).

11. J. M. Margolis, Conductive Polymers and Plastics, Chapman \& Hall, London. (1989).

12. W. F. Wu and B. S. Chiou, Thin Solid Films 298, 221 (1997).

13. Z. Yang, S. Han, T. Yang, L. Ye, H. Ma, and C. Cheng, Appl. Surf. Sci. 161, 279 (2000).

14. D. Kim and S. Kim, Thin Solid Films 408, 218 (2002).

15. K. N. Rao, Bull. Mater. Sci. 26, 239 (2003).

16. G. Frank, E. Kauer, and H. Kostlin, Thin Solid Films 77, 107 (1981).

17. L.-J. Meng, J. Gao, M. P. dos Santos, X. Wang, and T. Wang, Thin Solid Films 516, 1365 (2008).

Received: 15 February 2008. Accepted: 5 August 2008. 